\title{
A UNIVERSAL EXPONENT FOR HOMEOMORPHS
}

\author{
PETER KEEVASH, JASON LONG, BHARGAV NARAYANAN, AND ALEX SCOTT
}

\begin{abstract}
We prove a uniform bound on the topological Turán number of an arbitrary two-dimensional simplicial complex $\mathcal{S}$ : any $n$-vertex two-dimensional complex with at least $C_{\mathcal{S}} n^{3-1 / 5}$ facets contains a homeomorphic copy of $\mathcal{S}$, where $C_{\mathcal{S}}>0$ is an absolute constant depending on $\mathcal{S}$ alone. This result, a two-dimensional analogue of a classical result of Mader for one-dimensional complexes, sheds some light on an old problem of Linial from 2006.
\end{abstract}

\section{INTRODUCTION}

A number of natural extremal geometric problems arise when we view an $r$-uniform hypergraph as an $(r-1)$-dimensional simplicial complex (by identifying edges with facets). Questions of this nature arise in the high-dimensional combinatorics programme of Linial [12, 10], and have also been raised by Gowers [5]; for a sample of some recent results in this programme, see $[4,14,13,11]$. In this paper, we study the Turán problem for 2-complexes, or equivalently, the topological Turán problem for 3-graphs.

In the Turán theory of 3-graphs (see $[17,7]$ ), one is concerned with finding a copy of a fixed 3-graph as a subgraph; in the context of 2-complexes, the appropriate replacement for the notion of a subgraph is that of a homeomorphic image. More formally, we say that two 3-graphs $G$ and $H$ are homeomorphic if they are homeomorphic as topological spaces (when viewed as 2-complexes), and we say that $G$ contains a homeomorph of $H$ if there is a subgraph of $G$ homeomorphic to $H$. The following example may help clarify this point of view: a 3-graph $H$ is a homeomorph of the complete 3 -graph $K_{4}^{3}$ on four vertices (or equivalently, the two-dimensional sphere $S^{2}$ ) if we can place the vertices of $H$ on the sphere and then triangulate the sphere using those vertices in such a way that the resulting triangles are precisely the edges of $H$. In this language, our main contribution is the following theorem.

Theorem 1.1. For each 3-graph $H$, there exists $C_{H}>0$ such that any 3-graph $G$ on $n$ vertices with at least $C_{H} n^{3-1 / 5}$ edges contains a homeomorph of $H$.

Here, it is worth mentioning that the topological Turán problem for 2-graphs (i.e., graphs) is understood reasonably well: a classical result of Mader [15] asserts that for any graph $H$, there exists $C_{H}>0$ such that every $n$-vertex graph with at least $C_{H} n$ edges contains a homeomorph of $H$, and this is tight in general up to the multiplicative constant. Linial [8,9] has raised the question of an analogous result for 3-graphs, and while answers are available for a few specific 3-graphs, no general results for 3-graphs in the spirit of Mader's theorem appear to have been previously known; our main result, Theorem 1.1, fills in this gap.

We shall in fact prove Theorem 1.1 with $C_{H}=2000 v(H)^{6}$ for all sufficiently large $n \in \mathbb{N}$. However, we make no attempt to optimise this constant since we do not believe the exponent of $3-1 / 5$ in our result to be tight; instead, we expect the right exponent to be $3-1 / 2=5 / 2$, and make the following conjecture.

Date: 24 March, 2020.

2010 Mathematics Subject Classification. Primary 05E45; Secondary 05C65, 05C35. 


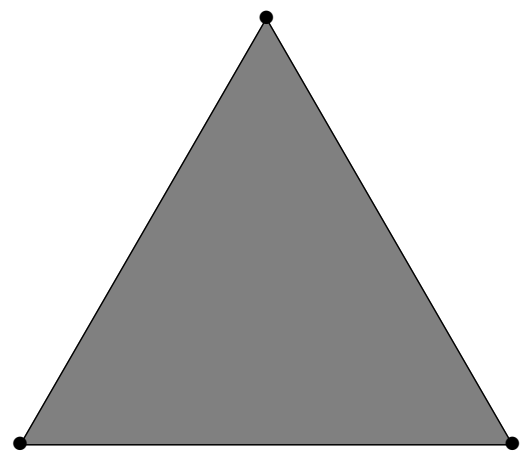

H

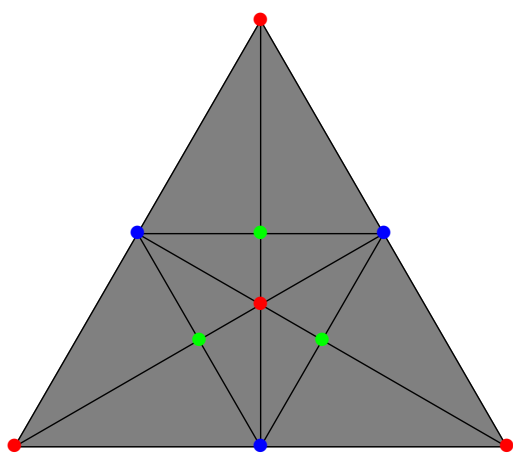

$\tilde{H}$

Figure 1. Each edge of $H$ maps to twelve new edges in $\tilde{H}$; the colours red, blue and green describe the tripartition of $\tilde{H}$.

Conjecture 1.2. For each 3-graph $H$, there exists $C_{H}>0$ such that any 3-graph $G$ on $n$ vertices with at least $C_{H} n^{5 / 2}$ edges contains a homeomorph of $H$.

This conjectural exponent of 5/2 requires explanation, and this brings us to the starting point of the line of investigation we pursue in this paper. In the specific case of the tetrahedron $K_{4}^{3}$, a classical result of Brown, Erdős and Sós [1] says that 5/2 is indeed the correct exponent: the minimum number of edges guaranteeing a homeomorph of the sphere in an $n$-vertex 3-graph is $\Theta\left(n^{5 / 2}\right)$. Conjecture 1.2 is then motivated by the following line of reasoning: it turns out that we may find homeomorphs in 2-graphs roughly once we are able to find cycles, i.e., homeomorphs of $S^{1}$, and our investigations suggest that we ought to be able to find homeomorphs in 3-graphs roughly once we are able to find spheres, i.e., homeomorphs of $S^{2}$.

The arguments of Brown, Erdős and Sós are however rather specific to the sphere and, slightly more generally, to 'double-pyramidal' complexes. Consequently, even the specialisation of Conjecture 1.2 to specific 3-graphs leads to interesting questions; indeed, the special case of $H$ being (a triangulation of) the torus remains open, and has been reiterated by Linial $[8,9]$ on multiple occasions as a natural starting point.

To put Theorem 1.1 in context, another (cheap) argument is worth mentioning: for any 3 -graph $H$, it is not difficult to construct a 3-partite 3-graph $\tilde{H}$ that is homeomorphic to $H$ (as shown in Figure 1 ), and since finding a copy of $\tilde{H}$ as a subgraph is a degenerate Turán problem, it follows from a classical result of Erdős [3] that there is an $\varepsilon_{H}>0$ such that any $n$-vertex 3 -graph with at least $n^{3-\varepsilon_{H}}$ edges contains a copy of $\tilde{H}$ as a subgraph, and hence a homeomorphic copy of $H$. In contrast, Theorem 1.1 says that this $H$-specific exponent $\varepsilon_{H}$ may actually be replaced by a universal exponent of $1 / 5$.

The level of generality at which Theorem 1.1 applies comes at a price, however: for a few specific 3-graphs of interest, such as the sphere and the torus for example, the aforementioned arguments (i.e., that of Brown-Erdös-Sós, and the one based on the degenerate 3-graph Turán problem) yield better estimates than what is promised by Theorem 1.1 .

This paper is organised as follows. We begin with some definitions and establish some of the basic notions we need for the proof of our main result in Section 2. The proof of Theorem 1.1 then follows in Section 3. We conclude with a discussion about the limits of our approach, as well as some open problems, in Section 4. 


\section{Preliminaries}

Our notation is for the most part standard. Given a 2-graph or a 3-graph $G$, we write $v(G)$ and $e(G)$ for the number of vertices and edges of $G$ respectively. For a set $S$ of vertices in a 2-graph $G$, we write $\Gamma(S)$ for the set of common neighbours of $S$ in $G$, and following a common abuse, we write $\Gamma(x)$ for $\Gamma(\{x\}), \Gamma(x, y)$ for $\Gamma(\{x, y\})$, and so on; in the sequel, whenever we refer to $\Gamma(\cdot)$, the underlying graph will always be clear from the context, so there should be no cause for confusion. In those arguments that will involve working with both 2-graphs and 3-graphs in close proximity, we shall refer to the edges of 3-graphs as faces to avoid confusion. Finally, in what follows, pairs and triples refer respectively to unordered two-element and three-element sets; again, we abuse notation slightly and abbreviate a pair $\{x, y\}$ as $x y$, a triple $\{x, y, z\}$ as $x y z$, and so on.

It will be convenient to work with 3 -partite 3 -graphs; the following fact facilitates this, and follows from an easy averaging argument.

Proposition 2.1. Any 3-graph on $3 n$ vertices with $m$ edges contains a 3-partite subgraph with vertex classes of size $n$ and at least $2 \mathrm{~m} / 9$ edges.

Now, let $H$ be a fixed 3-graph and let $G$ be a 3-partite 3-graph whose three vertex classes $X, Y$ and $Z$ are each of size $n$. Our strategy to construct a homeomorph of $H$ in $G$ will involve gluing various building blocks together appropriately; below, we introduce the notions we require to execute this strategy.

First, we shall construct an auxiliary 2-graph $\mathcal{S}(H)$ from $H$ that will be helpful in finding a homeomorphic copy of $H$ in $G$. The construction of $\mathcal{S}(H)$ from $H$, illustrated in Figure 2, is as follows: first, for each pair $x y$ that is contained in some face of $H$, we introduce a new vertex $u=u_{x y}$ in $\mathcal{S}(H)$ and add the edges $x u$ and $y u$ to $\mathcal{S}(H)$; then, for each face $x y z$ of $H$, we introduce a new vertex $u=u_{x y z}$ in $\mathcal{S}(H)$ and add the edges $x u, y u$ and $z u$ to $\mathcal{S}(H)$.

We record a few facts about $\mathcal{S}(H)$ below.

(1) Each face $x y z$ of $H$ gives rise to three specific 4-cycles in $\mathcal{S}(H)$, namely the 4-cycles $\left\{x, u_{x y}, y, u_{x y z}\right\}$, $\left\{y, u_{y z}, z, u_{x y z}\right\}$ and $\left\{z, u_{z x}, x, u_{x y z}\right\}$; we call the 4-cycles of this form the special 4-cycles of $\mathcal{S}(H)$.

(2) $\mathcal{S}(H)$ is bipartite, with the set $V_{1}$ of the original vertices of $H$ and the set $V_{2}$ of the new vertices added in the construction of $\mathcal{S}(H)$ forming a bipartition.

(3) The degree of any vertex of $\mathcal{S}(H)$ in $V_{2}$ is at most 3.

Next, we describe the structures within $G$ that will serve as building blocks in constructing a homeomorph of $H$. The link graph $\mathcal{L}_{z}$ of a vertex $z \in Z$ is the bipartite graph between $X$ and $Y$ whose edges are those pairs $x y$ for which $x y z$ is a face of $G$. Notice that a 4-cycle in the link graph $\mathcal{L}_{z}$ corresponds to four faces of $G$ (all sharing the vertex $z$ ) that, taken together, are homeomorphic to a disk; we call such a collection of four faces of $G$ a 4-disk with centre $z$, and call the associated 4-cycle in the link graph $\mathcal{L}_{z}$ the boundary of the 4-disk.

Notice that a fixed 4-cycle in the complete bipartite graph between $X$ and $Y$ may be the boundary of anywhere between 0 and $n$ different 4-disks in $G$. We set $K_{H}=3 v(H)^{3}$, and call a 4-cycle between $X$ and $Y$

(1) $H$-admissible if the cycle is the boundary of more than $K_{H}$ different 4-disks in $G$, and

(2) $H$-forbidden if this cycle is the boundary of between 0 and $K_{H}$ different 4-disks in $G$.

The definitions of admissible and forbidden 4-cycles are motivated by the following observation. As noted earlier, each face of $H$ corresponds to three special 4-cycles in $\mathcal{S}(H)$, and if we manage to find a copy of $\mathcal{S}(H)$ 


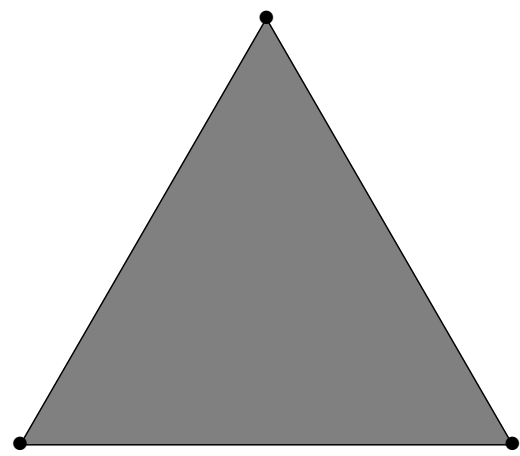

$H$

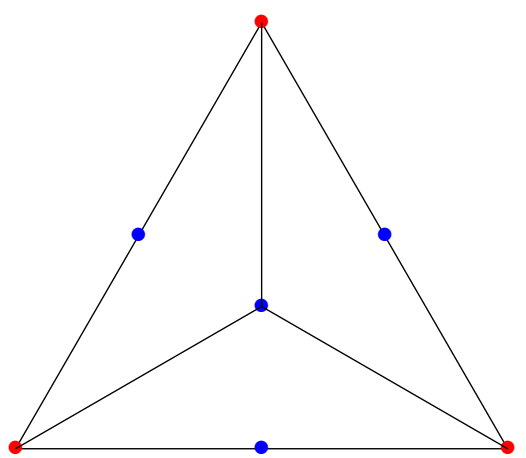

$\mathcal{S}(H)$

Figure 2. The construction of $\mathcal{S}(H)$ from $H$; the colours red and blue describe the bipartition of $\mathcal{S}(H)$.

in $X \times Y$ with the property that all its 4-cycles form boundaries of 4-disks in $G$ with distinct centres, then we may glue the corresponding 4-disks together to obtain a homeomorph of $H$ in $G$.

We shall rely on $H$-admissible 4-cycles between $X$ and $Y$ to build a homeomorph of $H$ in $G$. First, assuming $G$ has sufficiently many edges, we shall show that we may pass to a subgraph $G^{\prime}$ of $G$ in which most of the 4-cycles in $X \times Y$ are $H$-admissible. Next, we shall show, using $G^{\prime}$, that we may find a copy of $\mathcal{S}(H)$ between $X$ and $Y$ with the property that each of the 4-cycles in this copy is $H$-admissible. Finally, since an $H$-admissible 4-cycle is contained in at least $3 v(H)^{3} \geq 3 e(H)$ different link graphs, we will be able to ensure that we never re-use central vertices when gluing the appropriate 4-disks in $G^{\prime}$ together to construct a homeomorph of $H$.

\section{Proof of the main Result}

As before, let $H$ be a fixed 3-graph, take $K_{H}=3 v(H)^{3}$, and let $G$ be a 3-partite 3-graph whose three vertex classes $X, Y$ and $Z$ are each of size $n$.

Our first goal is to find a vertex $z \in Z$ whose link graph $\mathcal{L}_{z}$ is sufficiently dense so as to contain many copies of the auxiliary 2-graph $\mathcal{S}(H)$ defined in Section 2, and which has a small number of $H$-forbidden 4-cycles. In order to achieve this, we use a straightforward application of dependent random choice; with the set-up as above, we have the following claim.

Lemma 3.1. If $e(G) \geq C n^{3-\delta}$, then there exists a vertex $z \in Z$ such that

(1) $e\left(\mathcal{L}_{z}\right) \geq(C / 2) n^{2-\delta}$, and

(2) the number of $H$-forbidden 4 -cycles in $\mathcal{L}_{z}$ is at most $\left(2 K_{H} / C\right) n^{1+\delta} e\left(\mathcal{L}_{z}\right)$.

Proof. Select a vertex $z \in Z$ uniformly at random. It is clear that $\mathbb{E}\left[e\left(\mathcal{L}_{z}\right)\right]=e(G) / n$. Note that the probability that any given $H$-forbidden 4 -cycle is contained in $\mathcal{L}_{z}$ is at most $K_{H} / n$. Therefore, writing $B_{z}$ for the number of $H$-forbidden 4-cycles in $\mathcal{L}_{z}$, we have $\mathbb{E}\left[B_{z}\right] \leq K_{H} n^{3}$.

Putting the two estimates above together, we have

$$
\left.\mathbb{E}\left[e\left(\mathcal{L}_{z}\right)-(C / 2) n^{2-\delta}-\left(C / 2 K_{H}\right) n^{-1-\delta} B_{z}\right)\right] \geq 0,
$$


so there must exist a vertex $z \in Z$ for which we both have

$$
e\left(\mathcal{L}_{z}\right)-(C / 2) n^{2-\delta} \geq 0
$$

and

$$
e\left(\mathcal{L}_{z}\right)-\left(C / 2 K_{H}\right) n^{-1-\delta} B_{z} \geq 0,
$$

proving the claim.

Our proof of Theorem 1.1 will hinge around finding a copy of the auxiliary 2-graph $\mathcal{S}(H)$ within the link graph $\mathcal{L}_{z}$ promised by Lemma 3.1 while avoiding $H$-forbidden 4-cycles. To find this copy, we first show that we can pass to a large subset $Y^{\prime} \subset Y$ within which almost all pairs and triples are well-behaved. To quantify what it means to be well-behaved, we make the following definitions.

(1) We call a pair $y_{1} y_{2}$ of vertices in $Y$ good if

$$
\left|\Gamma\left(y_{1}, y_{2}\right)\right| \geq n^{1-2 \varepsilon}
$$

and there are at most

$$
\left(K_{H} / C\right) n^{1-3 \varepsilon}\left|\Gamma\left(y_{1}, y_{2}\right)\right|
$$

$H$-forbidden 4-cycles containing both $y_{1}$ and $y_{2}$, and bad otherwise.

(2) We call a triple $y_{1} y_{2} y_{3}$ of vertices in $Y$ good if

$$
\left|\Gamma\left(y_{1}, y_{2}, y_{3}\right)\right| \geq n^{1-3 \varepsilon},
$$

and bad otherwise.

With this set-up, we next show the following.

Lemma 3.2. Let $\varepsilon \leq 1 / 5, C \geq 1$ and let $\mathcal{L}_{z}$ be a bipartite graph between $X$ and $Y$ with $(C / 2) n^{2-\varepsilon}$ edges in which the number of $H$-forbidden 4 -cycles is at most $K_{H} n^{3+1 / 5-\varepsilon}$. Then there is a subset $Y^{\prime}$ of $Y$ of size at least $n^{1-\varepsilon} / 4$ within which

(1) at most $(400 / C)\left(\begin{array}{c}\left|Y^{\prime}\right| \\ 2\end{array}\right)$ pairs are bad, and

(2) at most $(600 / C)\left(\begin{array}{c}\left|Y^{\prime}\right| \\ 3\end{array}\right)$ triples are bad.

Proof. To prove the lemma, we start by selecting a vertex $x \in X$ uniformly at random. Clearly, we have

$$
\mathbb{E}[|\Gamma(x)|]=(C / 2) n^{1-\varepsilon} .
$$

Notice that if a pair $y_{1} y_{2}$ in $Y$ is bad, then either

(a) $\left|\Gamma\left(y_{1}, y_{2}\right)\right|<n^{1-2 \varepsilon}$, or

(b) the number of $H$-forbidden 4-cycles through $y_{1}$ and $y_{2}$ is at least $\left(K_{H} / C\right) n^{1-3 \varepsilon}\left|\Gamma\left(y_{1}, y_{2}\right)\right|$,

or possibly both.

First, given a bad pair $y_{1} y_{2}$ in $Y$ for which (a) holds, since $\left|\Gamma\left(y_{1}, y_{2}\right)\right|<n^{1-2 \varepsilon}$, the probability that both $y_{1}$ and $y_{2}$ belong to $\Gamma(x)$ is at most $n^{-2 \varepsilon}$; hence, the number $P_{1}$ of such pairs surviving in $\Gamma(x)$ satisfies

$$
\mathbb{E}\left[P_{1}\right] \leq n^{2-2 \varepsilon} .
$$


Next, let $\mathcal{Q}$ denote the set of bad pairs $y_{1} y_{2}$ for which (b) holds, so each pair $y_{1} y_{2} \in \mathcal{Q}$ lies in at least $\left(K_{H} / C\right) n^{1-3 \varepsilon}\left|\Gamma\left(y_{1}, y_{2}\right)\right| H$-forbidden 4 -cycles. Since the total number of $H$-forbidden 4 -cycles in $\mathcal{L}_{z}$ is at most $K_{H} n^{3+1 / 5-\varepsilon}$, we get

which implies that

$$
\sum_{y_{1} y_{2} \in \mathcal{Q}}\left(K_{H} / C\right) n^{1-3 \varepsilon}\left|\Gamma\left(y_{1}, y_{2}\right)\right| \leq K_{H} n^{3+1 / 5-\varepsilon}
$$

$$
\sum_{y_{1} y_{2} \in \mathcal{Q}}\left|\Gamma\left(y_{1}, y_{2}\right)\right| \leq C n^{2+1 / 5+2 \varepsilon}
$$

It follows that the number $P_{2}$ of pairs in $\mathcal{Q}$ surviving in $\Gamma(x)$ satisfies

$$
\mathbb{E}\left[P_{2}\right]=\sum_{y_{1} y_{2} \in \mathcal{Q}} \frac{\left|\Gamma\left(y_{1}, y_{2}\right)\right|}{n} \leq C n^{1+1 / 5+2 \varepsilon} .
$$

Thus, the total number $P_{x}$ of bad pairs surviving in $\Gamma(x)$, which is clearly at most the sum $P_{1}+P_{2}$, satisfies

$$
\mathbb{E}\left[P_{x}\right] \leq \mathbb{E}\left[P_{1}\right]+\mathbb{E}\left[P_{2}\right] \leq C n^{1+1 / 5+2 \varepsilon}+n^{2-2 \varepsilon} \leq(1+C) n^{2-2 \varepsilon} ;
$$

here, the last inequality relies on the fact that $\varepsilon \leq 1 / 5$.

Finally, given a bad triple $y_{1} y_{2} y_{3}$ in $Y$, since $\left|\Gamma\left(y_{1}, y_{2}, y_{3}\right)\right|<n^{1-3 \varepsilon}$, the probability that this triple survives in $\Gamma(x)$ is at most $n^{-3 \varepsilon}$. Writing $T_{x}$ for the number of bad triples surviving in $\Gamma(x)$, we again have

$$
\mathbb{E}\left[T_{x}\right] \leq n^{3-3 \varepsilon} .
$$

Putting the above estimates together, we get

$$
\mathbb{E}\left[|\Gamma(x)|-\frac{C n^{1-\varepsilon}}{4}-\frac{C P_{x}}{12(1+C) n^{1-\varepsilon}}-\frac{C T_{x}}{6 n^{2-2 \varepsilon}}\right] \geq 0,
$$

which in particular implies that there is some $x \in X$ for which we have

(A) $|\Gamma(x)| \geq C n^{1-\varepsilon} / 4$,

(B) $|\Gamma(x)| \geq C P_{x} / 12(1+C) n^{1-\varepsilon}$, and

(C) $|\Gamma(x)| \geq C T_{x} / 6 n^{2-2 \varepsilon}$.

Multiplying the inequality in (B) by the one in (A), the inequality in (C) by the square of the one in (A), we see that for this choice of $x \in X$, we have

$$
P_{x} \leq\left(48(1+C) / C^{2}\right)|\Gamma(x)|^{2} \leq(400 / C)\left(\begin{array}{c}
|\Gamma(x)| \\
2
\end{array}\right)
$$

and

$$
T_{x} \leq\left(96 / C^{3}\right)|\Gamma(x)|^{3}<\left(600 / C^{3}\right)\left(\begin{array}{c}
|\Gamma(x)| \\
3
\end{array}\right),
$$

provided $n$ is sufficiently large. Taking $Y^{\prime}=\Gamma(x)$ for this choice of $x$ proves the claim.

We are now ready to put these two lemmas together to prove our main result.

Proof of Theorem 1.1. Our goal given is to find a homeomorph of a given 3-graph $H$ in any large 3-graph $G$ with sufficiently many faces. Appealing to Proposition 2.1, we start by assuming that $G$ is a 3-partite 3 -graph whose three vertex classes $X, Y$ and $Z$ are each of size $n$, and which has at least $C n^{3-1 / 5}$ faces for some suitably large constant $C$ depending on $H$ alone. As described in Section 2, we shall work with the auxiliary 2-graph $\mathcal{S}(H)$ to find a homeomorph of $H$ in $G$. 
First, we apply Lemma 3.1 to $G$ with $\delta=1 / 5$. This gives us a vertex $z \in Z$ whose link graph $\mathcal{L}_{z}$ contains $(C / 2) n^{2-\varepsilon}$ edges for some $\varepsilon \leq \delta=1 / 5$ in which the number of $H$-forbidden 4-cycles is at most

$$
\left(2 K_{H} / C\right) n^{1+1 / 5} e\left(\mathcal{L}_{z}\right)=K_{H} n^{3+1 / 5-\varepsilon} .
$$

This link graph satisfies the requirements of Lemma 3.2, so we apply the lemma to pass to a subset $Y^{\prime} \subset Y$ within which most pairs and most triples are good.

Recall that $\mathcal{S}(H)$ is bipartite and admits a bipartition $\left(V_{1}, V_{2}\right)$ where each vertex in $V_{2}$ has degree at most 3 , and where $V_{1}$ is in fact the original set of vertices of $H$.

We shall first embed the vertices of $V_{1}$ into $Y^{\prime}$ in such a way that no embedded pair is bad and no embedded triple is bad. In order to show that this is possible, we note that the proportion of bad pairs in $Y^{\prime}$ is at most $400 / C$ and the proportion of bad triples in $Y^{\prime}$ is at most $600 / C^{3}$. We define a 3 -graph $\mathcal{D}\left(Y^{\prime}\right)$ on the vertex set $Y^{\prime}$ whose edges are those that are potentially problematic for our embedding, i.e., those triples $y_{1} y_{2} y_{3}$ which are either bad, or for which one of the pairs $y_{1} y_{2}, y_{2} y_{3}$ or $y_{1} y_{3}$ is bad. The density of this 3-graph $\mathcal{D}\left(Y^{\prime}\right)$ is at most $1200 / C+600 / C^{3} \leq 2000 / C$.

Our goal now is to find a complete 3 -graph on $\left|V_{1}\right|$ vertices in the complement of $\mathcal{D}\left(Y^{\prime}\right)$, since the existence of such a subgraph enables us to inject $V_{1}$ into $Y^{\prime}$ whilst avoiding all bad pairs and bad triples. A bound of de Caen [2] shows that a copy of the complete 3-graph $K_{t}^{3}$ on $t$ vertices can be found in any 3-graph on $n$ vertices of density at least

$$
1-\left(\begin{array}{c}
t-1 \\
2
\end{array}\right)^{-1}
$$

provided $n$ is sufficiently large. Therefore, we may find our embedding (again, assuming that $n$ is sufficiently large) provided that

$$
2000 / C \leq\left(\begin{array}{c}
\left|V_{1}\right| \\
2
\end{array}\right)^{-1}
$$

which we may ensure by taking $C \geq 1000 v(H)^{2}$.

It remains to find an embedding of the vertices of $V_{2}$ into $X$. For each vertex $u$ of degree 3 in $V_{2}$ that we need to embed into $X$, we have a choice of $n^{1-3 \varepsilon}$ vertices in the common neighbourhood of its three already-embedded neighbours from $V_{1}$; we choose its image from these candidates uniformly at random. Similarly, for each vertex $v$ of degree 2 in $V_{2}$, we choose its image uniformly at random from the $n^{1-2 \varepsilon}$ vertices in the common neighbourhood of its two already-embedded neighbours from $V_{1}$.

The probability that this embedding is not proper, i.e., that some two vertices in $V_{2}$ get mapped to the same vertex in $X$, is at most $\left|V_{2}\right|^{2} n^{3 \varepsilon-1}<1 / 2$, provided $n$ is large (since $\varepsilon \leq 1 / 5$ and $\left|V_{2}\right| \leq 10 v(H)^{3}$ ).

We shall next show that for this embedding, the probability of some special 4-cycle in $\mathcal{S}(H)$ mapping to an $H$-forbidden 4 -cycle is also at most $1 / 2$. Since the number of special 4-cycles in $\mathcal{S}(H)$ is $3 e(H)$, it suffices to show for each special 4-cycle $\mathcal{C}$ in $\mathcal{S}(H)$ that the probability of its image being $H$-forbidden is at most $1 /(6 e(H))$. Let $y_{1}$ and $y_{2}$ be the images of vertices of $\mathcal{C}$ in $V_{1}$ (which have been fixed earlier deterministically), and consider $u^{\prime}$ and $v^{\prime}$, the (random) images of the two vertices $u$ and $v$ of $\mathcal{C}$ from $V_{2}$ whose degrees in $\mathcal{S}(H)$ are respectively 3 and 2 . Let $y_{3}$ be the vertex in $Y^{\prime}$ so that $u^{\prime}$ is chosen uniformly at random from $\Gamma\left(y_{1}, y_{2}, y_{3}\right)$. Suppose for a contradiction that the probability of the image of $\mathcal{C}$ being $H$-forbidden is at least $1 /(6 e(H))$. Then this implies that at least a $1 /(6 e(H))$ proportion of the 4-cycles formed by taking $y_{1}$ and $y_{2}$, together with a vertex $x_{1} \in \Gamma\left(y_{1}, y_{2}, y_{3}\right)$ and a vertex $x_{2} \in \Gamma\left(y_{1}, y_{2}\right)$ are $H$-forbidden. This leads us to conclude that the number of $H$-forbidden 4-cycles through $y_{1}$ and $y_{2}$ is at least

$$
(1 / 6 e(H)) n^{1-3 \varepsilon}\left|\Gamma\left(y_{1}, y_{2}\right)\right| \text {. }
$$


However, if $1 /(6 e(H)) \geq K_{H} / C$, then this would imply that $y_{1} y_{2}$ is a bad pair, a contradiction; this final inequality may be ensured by taking $C \geq 18 v(H)^{6}$, since $e(H) \leq v(H)^{3}$ and $K_{H}=3 v(H)^{3}$.

We have shown that it is possible to embedded $\mathcal{S}(H)$ into $\mathcal{L}_{z}$ in such a way that all of the special 4-cycles in this embedding are $H$-admissible. This embedding extends to a homeomorph of $H$ inside $G$ as follows. For each 4-cycle $\mathcal{C}$ in $\mathcal{L}_{z}$ that is the image of some special 4-cycle of $\mathcal{S}(H)$, we claim that we may choose a unique vertex $z(\mathcal{C}) \in Z$ such that $\mathcal{C}$ is also contained in the link graph $\mathcal{L}_{z(\mathcal{C})}$ : indeed, $\mathcal{C}$ is $H$-admissible, so there are at least $K_{H}=3 v(H)^{3} \geq 3 e(H)$ choices for $z(\mathcal{C})$. We then use $z(\mathcal{C})$ to turn each of the embedded special 4-cycles $\mathcal{C}$ in $\mathcal{L}_{z}$ into a 4-disk in $G$, noting that these 4-disks all have distinct centres; the result is a homeomorph of $H$ in $G$.

\section{Conclusion}

Below, we address some of the limitations of our approach to finding homeomorphs of a fixed target 3 -graph $H$, as well as some potential avenues for improvement.

It seems plausible that the exponent of $3-1 / 5$ that we obtain may be improved somewhat by a more judicious application of the methods developed here. However, the ideas developed in this paper reach a bottleneck, conjecturally, at the exponent of $3-1 / 4$. This is because it is believed [16] that there exist $n$-vertex 3 -graphs with $\Omega\left(n^{3-1 / 4}\right)$ edges that do not contain any octahedra, though the best constructions presently known, see [6], only manage $\Omega\left(n^{3-1 / 3}\right)$ edges. If a 3-graph does not contain any octahedra, then our approach based on $H$-admissible 4 -cycles falls apart, since if all the 4 -cycles in the link graphs are $H$-forbidden, then our method for extending $\mathcal{S}(H)$ to a homeomorph of $H$ fails due to degeneracy concerns.

Another important fact to bear in mind is that while $\Omega\left(n^{5 / 2}\right)$ edges guarantee a homeomorph of $S^{2}$ in any $n$-vertex 3-graph, the number of edges needed to guarantee a homeomorph of $S^{2}$ of bounded size comes with an exponent strictly greater than $5 / 2$, as can be verified by a standard deletion argument applied to a (binomial) random 3-graph of the appropriate density. Our methods here end up finding bounded-size homeomorphs: indeed, we find a homeomorphic copy of $H$ that has $12 e(H)$ edges. Any strategy that does not plan for the possibility of finding large homeomorphs of $H$, i.e., of size unbounded in terms of $H$, cannot prove Conjecture 1.2.

We leave the reader with a reminder of the specialisation of Conjecture 1.2 to the torus as reiterated by Linial $[8,9]$.

Conjecture 4.1. There is a $C>0$ such that any 3 -graph $G$ on $n$ vertices with at least $C n^{5 / 2}$ edges contains a homeomorph of the torus.

An easy adaptation of the arguments of Brown, Erdős, and Sós [1] to triple-pyramidal complexes (from double-pyramidal complexes) shows that an exponent of $3-1 / 3$ suffices for the torus, but improving on this bound remains an attractive starting point to Conjecture 1.2 in its full generality.

\section{ACKNOWLEDGEMENTS}

The first and second authors were partially supported by ERC Consolidator Grant 647678, and the third author wishes to acknowledge support from NSF grant DMS-1800521. 


\section{REFERENCES}

1. W. G. Brown, P. Erdős, and V. T. Sós, On the existence of triangulated spheres in 3-graphs, and related problems, Period. Math. Hungar. 3 (1973), 221-228.

2. D. de Caen, Extension of a theorem of Moon and Moser on complete subgraphs, Ars Combin. 16 (1983), $5-10$.

3. P. Erdős, On extremal problems of graphs and generalized graphs, Israel J. Math. 2 (1964), 183-190.

4. A. Georgakopoulos, J. Haslegrave, R. Montgomery, and B. Narayanan, Spanning surfaces in 3-graphs, Preprint, arXiv:1808.06864.

5. W. T. Gowers, Personal communication.

6. N. H. Katz, E. Krop, and M. Maggioni, Remarks on the box problem, Math. Res. Lett. 9 (2002), 515-519.

7. P. Keevash, Hypergraph Turán problems, Surveys in Combinatorics 2011, London Math. Soc. Lecture Note Ser., vol. 392, Cambridge Univ. Press, Cambridge, 2011, pp. 83-139.

8. N. Linial, What is high-dimensional combinatorics?, Random-Approx (2008).

9. __ Challenges of high-dimensional combinatorics, Lovász's Seventieth Birthday Conference (2018).

10. N. Linial and Z. Luria, An upper bound on the number of high-dimensional permutations, Combinatorica 34 (2014), 471-486.

11. _ Discrepancy of high-dimensional permutations, Discrete Analysis (2016:11), 8 pp.

12. N. Linial and A. Morgenstern, On high-dimensional acyclic tournaments, Discrete Comput. Geom. 50 (2013), 1085-1100.

13. N. Linial and Y. Peled, On the phase transition in random simplicial complexes, Ann. of Math. 184 (2016), 745-773.

14. Z. Luria and R. J. Tessler, A sharp threshold for spanning 2-spheres in random 2-complexes, Proc. Lond. Math. Soc. 119 (2019), 733-780.

15. W. Mader, Homomorphieeigenschaften und mittlere Kantendichte von Graphen, Math. Ann. 174 (1967), 265-268.

16. D. Mubayi, Some exact results and new asymptotics for hypergraph Turán numbers, Combin. Probab. Comput. 11 (2002), 299-309.

17. P. Turán, On an extremal problem in graph theory, Matematikai és Fizikai Lapok, 436452.

Mathematical Institute, University of Oxford, Andrew Wiles Building, Radcliffe Observatory Quarter, WoOdstock RoAd, OxFord OX2 6GG, UK

E-mail address: keevash@maths.ox.ac.uk

Mathematical Institute, University of Oxford, Andrew Wiles Building, Radcliffe Observatory Quarter, WOODSTOCK ROAD, OxFord OX26GG, UK

E-mail address: jlong@maths.ox.ac.uk

Department of Mathematics, Rutgers University, Piscataway, NJ 08854, USA

E-mail address: narayanan@math.rutgers.edu

Mathematical Institute, University of Oxford, Andrew Wiles Building, Radcliffe Observatory Quarter, WoOdsTOCK ROAD, OXFORd OX2 6GG, UK

E-mail address: scott@maths.ox.ac.uk 